\title{
Decision algorithm and surgical strategies for managing tracheocutaneous fistula
}

\author{
Chieh-Ni Kao $^{1 \#}$, Yu-Wei Liu ${ }^{2 \#}$, Po-Chih Chang ${ }^{2,3}$, Shah-Hwa Chou ${ }^{2,3}$, Su-Shin Lee ${ }^{1,3,4}$, Yur-Ren Kuo ${ }^{1,3,4}$, \\ Shu-Hung Huang ${ }^{1,3,4}$
}

${ }^{1}$ Division of Plastic Surgery, Department of Surgery, Kaohsiung Medical University Hospital, ${ }^{2}$ Division of Thoracic Surgery, Department of Surgery, Kaohsiung Medical University Hospital, ${ }^{3}$ Department of Surgery, School of Medicine, College of Medicine, ${ }^{4}$ Regeneration Medicine and Cell Therapy Research Center, Kaohsiung Medical University, Kaohsiung

Contributions: (I) Concept and design: CN Kao, YW Liu, Huang SH; (II) Administrative support: SS Lee, YR Kuo; (III) Provision of study materials or patients: CN Kao, YW Liu, PC Chang, SH Chou, SS Lee, YR Kuo, SH Huang; (IV) Collection and assembly of data: CN Kao, YW Liu; (V) Data analysis and interpretation: CN Kao, YW Liu, SH Huang; (VI) Manuscript writing: All authors; (VII) Final approval of manuscript: All authors. \#These authors contributed equally to this work.

Correspondence to: Shu-Hung Huang, MD, PhD. Division of Plastic Surgery, Department of Surgery, Kaohsiung Medical University Hospital, Kaohsiung Medical University, No. 100, Tzyou 1st Road, Kaohsiung. Email: huangsh63@gmail.com.

Background: Persistent tracheocutaneous fistula (TCF) is a complication of prolonged use of tracheostomy tube. Although many procedures exist to correct this issue, there is no consensus regarding its optimal management. We constructed a decision algorithm to determine appropriate surgical strategies for TCF repair.

Methods: Retrospectively reviewing our hospital's records, we found fourteen consecutive patients who had received surgical repair of tracheocutaneous fistula (primary closure or advanced local flap) between February 2013 and December 2018 and collected data relevant to their cases.

Results: We identified 11 male and 3 female patients. Duration of tracheostomy dependence was $8.1 \pm 4.7$ months, and timespan from decannulation to surgical closure $7.4 \pm 6.5$ months. Seven patients received primary closures, six received hinged turnover flaps, and one received random and perforator flap reconstruction. There was no perioperative mortality or morbidity except for one patient requiring a repeat tracheostomy 11 months after TCF repair due to pneumonia and subsequent respiratory failure. We used our findings and those reported in the literature to construct a modified risk factor scoring system based on patient's physical status, major comorbidities, perifistular soft tissue condition, and nutritional status and an algorithm for managing TCF based on the patients' fistula size and modified risk factor scores.

Conclusions: In conclusion, we were able to review our cases and those of other studies to create a risk scoring system and a decision algorithm that we believe will help optimize patient-directed surgical management of TCF repair.

Keywords: Tracheocutaneous fistula; tracheostomy; surgery; strategy; algorithm

Submitted Nov 15, 2019. Accepted for publication Dec 16, 2019.

doi: $10.21037 /$ jtd.2020.01.08

View this article at: http://dx.doi.org/10.21037/jtd.2020.01.08

\section{Introduction}

Tracheostomies are frequently performed during the treatment of critically ill patients in intensive care (1). Decannulation methods vary depending on a patient's ability to tolerate certain procedures and physician's experience. In most cases, a simple removal of the tube and the application of occlusive dressings produce acceptable functional and aesthetic results (2). However, the procedure is performed with some risk of subsequent development of tracheocutaneous fistulas (TCF), which develop when 
the squamous epithelium migrates into the trachea creating an epithelialized tract that fails to close. Kulber et al. have reported that $70 \%$ of adult patients who have tracheostomies for more than 16 weeks end up with TCFs (3). It has also been reported that $13 \%$ to $43 \%$ of children with tracheostomies develop TCFs after decannulation (4). Prolonged cannulation can significantly increase the risk of persistent fistulas, which can then lead to difficulties in vocalization and secretion clearance, and recurrent respiratory infections as well as social and cosmetic problems (5). Thus, TCFs often require local wound care and plastic reconstruction.

Our review of the literature revealed that TCFs can be corrected using several surgical techniques, including electrocautery (4), primary closure with or without fistulectomy $(5,6)$, use of a hinged turnover flap or an additional V-Y advancement flap (7-9), and harvest of auricular or rib cartilage transplanted to the tracheal defect with muscle and fasciocutaneous flaps (10-12). Each procedure has its own advantage and is used depending on individual patient needs and TCF characteristics, and choice of which procedure to use largely depends on expert opinion. However, we found very little consensus regarding the optimal management of TCF. Therefore, although cases are rare in number, our team set out to create an algorithm to help us decide on the most appropriate surgical strategy for repairing TCF based on the characteristics of previous cases reported in the literature and a retrospective review of cases at our hospital from 2013 to 2018.

\section{Methods}

\section{Study design and patients}

This retrospective study was approved by the institutional review board of Kaohsiung Medical University Hospital [approval number: KMUHIRB-E(I)-20190146]. Because the study was retrospective in design, the need for informed consent was waived. Our patient cases, mostly treated in our thoracic surgery and plastic surgery divisions, were referred to us for surgical repair of TCF if spontaneous closure of the fistula had not occurred after at least three months after decannulation of tracheostomy tubes or if the patient requested help resolving an unsightly cosmesis, weak cough, recurrent skin irritation related to draining secretions, or constraints on their daily activities.

Searching our hospital's medical records, we identified fourteen patients that had received TCF repairs between
February 2013 and December 2018 at our hospital. We collected and analyzed several clinically-relevant variables, including demographic characteristics, precipitating factors for tracheostomy, duration of tracheostomy dependence, timespan between cannulation to surgical closure, preoperative assessment, fistula size, operative techniques, operative time, and hospital length of stay. Prior to TCF repair, fiberoptic bronchoscopy was used to evaluate each patient's airway to ensure there were no tracheal abnormalities, such as tracheomalacia or granulation growth, that may need to be resolved before initiating surgery. Computed tomography of the neck or chest as well as preoperative overnight oximetry with the fistula occlusion test was selectively performed based on individual need. All the patients received surgical repair of TCF either under local anesthesia or general anesthesia depending on which, according to surgeon's clinical judgment, would be most appropriate for fistula size and patient condition. After surgery, all patients were advised to return for regular outpatient clinic visits. All of the cases were followed until January 2019.

\section{Representative TCF repair under general anesthesia}

\section{Patient No. 8}

Case 8 involved a 33-year-old man who had been dependent on tracheostomy for 16 months following a traumatic brain injury. After decannulation, a TCF had developed and was found to remain at an unreduced size of $10-\mathrm{mm}$ at 14 months (Figure 1A). The patient sought TCF repair because he had great difficulty speaking, coughing, and showering.

Because the patient's persistent TCF was large and might require more complex procedures, it was decided the TCF would be performed under general anesthesia. The patient was placed in a supine position with neck extended and intubated using a single endotracheal tube. One spindle-shaped incision was made around the TCF. The surgeon prepared three skin flaps to cover the skin defect: two hinge flaps for the right and left side of the fistula, respectively, and a third flap (advanced flap) to the right of the hinge flaps to eventually cover the whole area. Dissection proceeded through to the peritracheal area, preserving an area of fibrotic subcutaneous tissue around the fistula (Figure 1B). The hinged turnover flaps were sutured with 4-0 polydioxanone to make an inner lining for the trachea to cover the tracheal lumen with skin epithelium (Figure 1C). The subcutaneous tissues around the fistula were then sutured with 4-0 vicryl, using multiple layered 

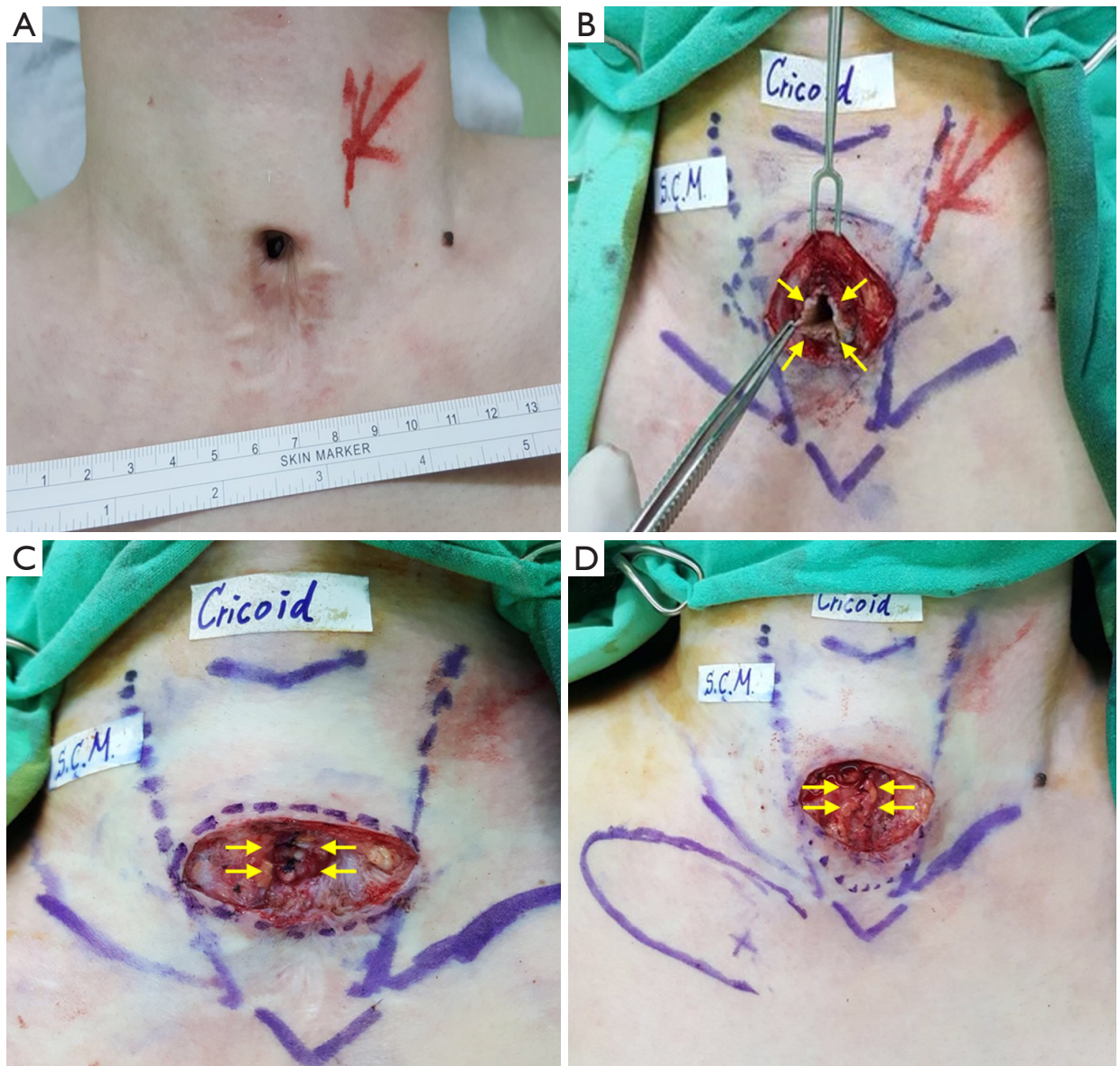

Figure 1 Surgical repair for Patient No.8 with tracheocutaneous fistula. (A) The TCF size was measured around 10 mm. (B) Dissection proceeded through to the peritracheal area, preserving an area of fibrotic subcutaneous tissue around the fistula (yellow arrows). (C) The hinged turnover flaps were sutured with 4-0 polydioxanone to make an inner lining for the trachea to cover the tracheal lumen with skin epithelium (yellow arrows). (D) The subcutaneous tissues around the fistula were then sutured with 4-0 vicryl using multiple layered sutures to invaginate the hinged flaps into the fistula (yellow arrows).

sutures to invaginate or embed the hinged flaps into the fistula (Figure 1D). Following flap design and identification of perforator by hand-held doppler, a perforator-based transposition flap was rotated into the closed fistula, fixed, and finally closed with 5-0 nylon (Figure 2A,B). Figure $2 C$ shows the wound 1 month after surgery during follow-up in an outpatient clinic.

\section{Representative TCF repair under local anesthesia}

\section{Patient No. 9}

Case 9 involved a 54-year-old woman who had been dependent on tracheostomy for 6 months after a traumatic brain injury. After decannulation, she was found to have developed an TCF. Eight months later, although the size of the fistula had reduced somewhat, the patient requested TCF repair to improve its appearance and reduce build-up of secretions around it. At that time, it had reduced to $<5-\mathrm{mm}$ in size (Figure $3 \mathrm{~A}$ ). Surgical repair was performed much as it was performed in Patient 8 mentioned above. Fistula and the surrounding skin were injected within $2 \%$ lidocaine combined with 1:100 epinephrine, to a total volume of less than $10 \mathrm{~mL}$. One smaller spindle-shaped incision was made around the TCF after identification via needlestick probe and routine lidocaine spray through the probe was performed to reduce the cough reflex and secretion (Figure 3B). Three skin flaps were prepared (two hinge flaps on the right and left sides of the fistula and a third flap to the right of the hinge flaps) to cover the fistula. Then, dissection continued down to the peritracheal area, preserving an 

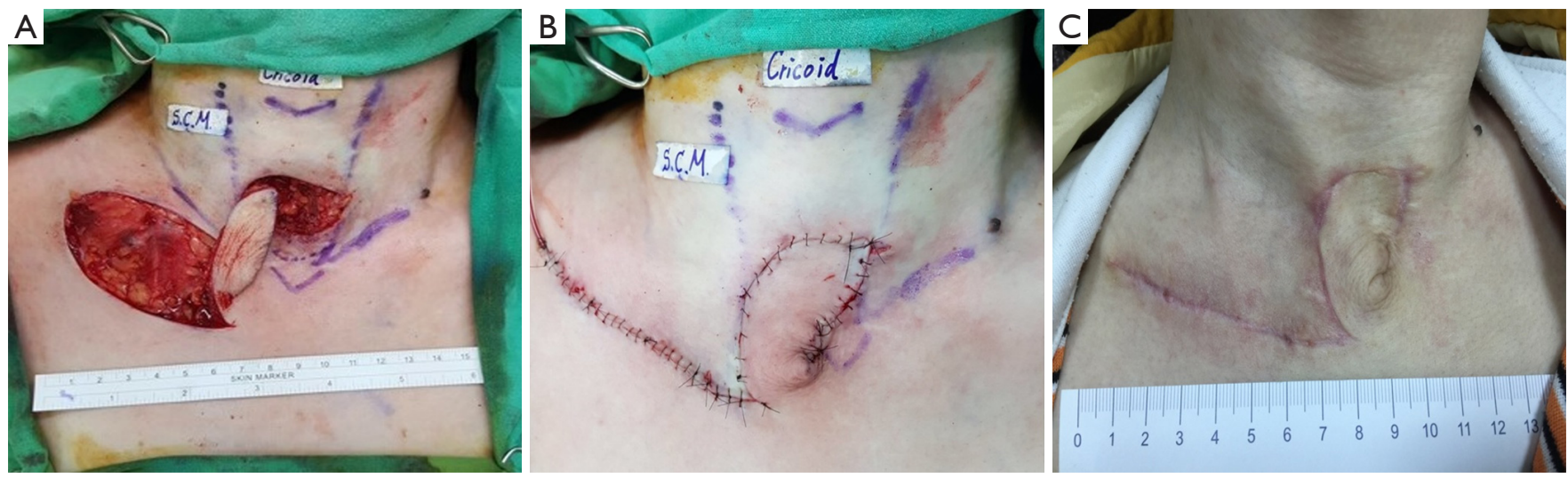

Figure 2 Surgical repair for Patient No.8 with TCF and postoperative follow-up wound condition. (A) Following flap design and identification of perforator by hand-held doppler, a perforator-based transposition flap was rotated into the closed fistula and fixed. (B) Wound closed with 5-0 nylon. (C) Day 30 postoperative wound.

area of fibrotic subcutaneous tissue around the fistula (Figure 3C). At this stage, however, the fistula was measured to determine whether a fistulectomy due to redundancy would be necessary. In this case, fistulectomy was performed. The hinged turnover flaps were sutured with 4-0 polydioxanone to make an inner lining for the trachea to cover the tracheal lumen with skin epithelium (Figure 3D). The subcutaneous tissues around the fistula were then sutured with 4-0 vicryl, using layered sutures to invaginate the hinged flaps into the fistula followed by wound closure with 5-0 nylon.

\section{Risk stratification scoring system}

In addition to the length of time with tracheostomy, there are other factors related to risk of persistent fistula, including age, radiation, history of previous tracheotomy, and obesity $(13,14)$. We searched the literature regarding the risk factors related to wound healing $(15,16)$ and reviewed our cases to identify factors potentially contributing to the formation of TCFs, and found reported contributing factors to fall within four categories: patient's physical status, major comorbidity, perifistular soft tissue condition, and nutritional status. We therefore construct a tentative risk stratification scoring system (Table 1).

\section{Statistical analysis}

Patient characteristics were analyzed descriptively and expressed as mean \pm standard deviation. All statistical operations were performed using SPSS 20 (SPSS Inc., Chicago, IL, USA) for Windows.

\section{Results}

The cases we identified at our hospital consisted of eleven male and three female patients aged 58.6 \pm 19.0 years. Prolonged mechanical ventilation necessitating tracheostomies were needed in five patients who had brain injuries incurred in motor vehicle collisions, eight patients who had complications related to surgical operations, and one who had an inhalation injury. Mean duration of tracheostomy dependence was $8.1 \pm 4.7$ months, and mean time interval from decannulation to surgical of TCF $7.4 \pm 6.5$ months. Simple primary closures with fistulectomies were performed in seven, hinged turnover flap with fistulectomies in six, and perforator flap reconstruction in one tailored to the fistula size and perifistular soft tissue condition. Mean operative time was $38.6 \pm 15.6$ minutes and mean post-operative hospital length of stay $2.3 \pm 2.2$ days (Table 2).

All patients had uneventful recoveries. During the followup period, which ranged from 1 to 71 months, there were no surgery-related complications or incomplete closures of TCF. However, one patient required a repeat tracheostomy 11 months after TCF repair due to pneumonia and subsequent respiratory failure necessitating prolonged ventilator support. The remaining thirteen patients has had complete fistula closures with no complications recorded during the follow-up period.

According to our constructed risk stratification scoring system (Table 1) in which a score of $0-3$ was assigned to low-risk patients for whom conservative treatment without additional surgical care would be safe and adequate, a score of 4-6 was assigned to intermediate-risk patients needing 

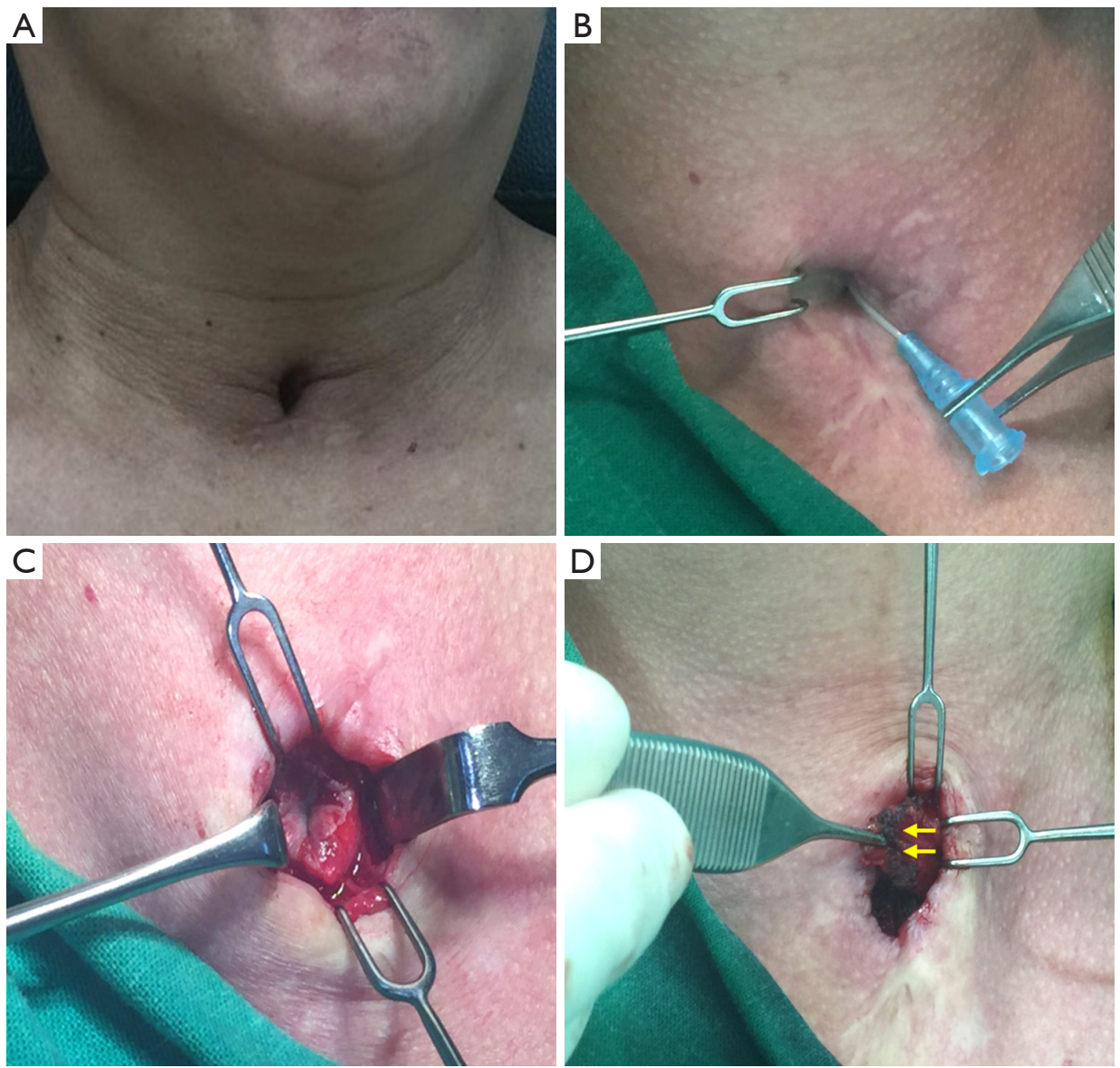

Figure 3 Surgical repair for patient No. 9 with tracheocutaneous fistula. (A) The TCF measured $<5$ mm. (B) TCF was identified via needlestick probe insertion followed by routine lidocaine spray through the probe and creation of a smaller spindle-shaped incision around the TCF. (C) The fistula was observed and fistulectomy was performed after dissection through to the peritracheal area. (D) The hinged turnover flaps were sutured with 4-0 polydioxanone to make an inner lining for the trachea to cover the tracheal lumen with skin epithelium (yellow arrows).

close observation and further evaluation before treatment plan is decided, and score of $7-10$ was assigned to high-risk patients with an increased rate of persistent fistula needing additional evaluation and/or surgical intervention. Based on this system, eight of our fourteen patients could be considered low-risk patients, five intermediate-risk patients, and one a high-risk patient.

\section{Discussion}

Persistent tracheocutaneous fistula is not uncommon following prolonged cannulations of tracheostomy. The duration of cannulation and a history of multiple tracheostomies are known to increase risk of persistent tracheocutaneous fistulas. Extending the duration of cannulation allows time for epithelial tissue to grow within the stoma and form epithelialized scar tissue and dense connective tissue, which results in persistent stoma. Fifty percent of patients cannulated for more than a year after a tracheostomy develop persistent tracheocutaneous fistulas (4).

Surgery remains a mainstay treatment for TCF repair. A wide array of surgical techniques for closing TCF have been used and reported to have favorable outcomes. They range from simple use of primary closure to use of a hinged turnover flap and even to use of a harvested auricular or rib cartilage to repair the large fistula defect $(4-7,9-12,14,17)$. Despite the great number of procedures that have been used, it remains unclear what optimal management of TCF would look like given the variety of scenarios. 
Table 1 Risk stratification scoring system

\begin{tabular}{|c|c|}
\hline Category & Score \\
\hline \multicolumn{2}{|l|}{ Patient's physical status } \\
\hline Elderly $>65$-year-old & 1 \\
\hline ECOG: 2-4 & 1 \\
\hline \multicolumn{2}{|l|}{ Major comorbidity } \\
\hline Poorly controlled diabetes mellitus* & 1 \\
\hline End-stage renal disease with hemodialysis & 1 \\
\hline Head \& neck cancer history & 1 \\
\hline \multicolumn{2}{|l|}{ Perifistular soft tissue condition } \\
\hline Previous tracheostomy & 1 \\
\hline Severe peristomal scarring & 1 \\
\hline Prior radiation of the neck & 1 \\
\hline \multicolumn{2}{|l|}{ Nutritional status } \\
\hline Obesity (BMI >30) & 1 \\
\hline Hypoalbuminemia (albumin <3.5) & 1 \\
\hline \multicolumn{2}{|l|}{ Risk stratification and scoring } \\
\hline $\begin{array}{l}\text { Low-risk patients: appropriate and safe for } \\
\text { conservative treatment without additional } \\
\text { surgical care }\end{array}$ & $0-3$ \\
\hline $\begin{array}{l}\text { Intermediate-risk patients: needing close } \\
\text { observation and further evaluation }\end{array}$ & $4-6$ \\
\hline $\begin{array}{l}\text { High-risk patients: associated with an } \\
\text { increased rate of persistent fistula needing } \\
\text { additional evaluation and/or surgical } \\
\text { intervention }\end{array}$ & $7-10$ \\
\hline
\end{tabular}

*Poorly controlled DM defined as an uninterrupted Hemoglobin A1c $(\mathrm{HbA} 1 \mathrm{c}) \geq 8.0 \%$ for $\geq 1$ year despite standard care. ECOG, Eastern Cooperative Oncology Group Performance Status; BMI, body mass index.

Although cases are few in number, we constructed a decision algorithm (Figure 4) for managing TCF based on fistula size and above-mentioned risk factors. After at least 3 months follow-up, patients with TCF are reassessed and further divided into group A (TCF size $<5 \mathrm{~mm}$ with spontaneous closure); group B (TCF size $<5 \mathrm{~mm}$ without spontaneous closure); group C (TCF size 5-10 mm); and group D (TCF size $>10 \mathrm{~mm}$ ). Group A consists of patients with small TCFs $(<5 \mathrm{~mm})$ after decannulation, all ultimately healing spontaneously within the observation time period. Groups B and C consisted of those with small TCFs $(<5 \mathrm{~mm})$ without spontaneous closure suitable for primary closure and those with fistula sizes $5-10 \mathrm{~mm}$ suitable for procedures in which the local flap technique is performed. There is no consensus in the literature regarding the best recommended surgical strategies for fistulas of different sizes. However, there are some case studies that report that patients with the TCFs larger than $10 \mathrm{~mm}$, those with poor skin elasticity, those with deep retraction of lateral tracheal walls, and post-radiation of the neck may require more complex surgical techniques, including transplantation of an ear or rib cartilage graft and tracheal stent placement (10-12). These were performed to ensure complete closure of the fistula, which may not be achieved by turnover flaps, muscular interpositions, or free flaps alone (7-9). Patients with large TCFs $(>10 \mathrm{~mm}$ ) were categorized into a group D, patients who might be in need of such complex procedures.

Five of our fourteen patients had small TCFs (size: $<5 \mathrm{~mm}$ ) and received fistula repairs under local anesthesia. They all recovered uneventfully and were discharged on the same day. Likewise, other studies have reported the successful TCF repair under local anesthesia. For example, Khaja et al. reported using primary multilayer closure under local anesthesia to successfully repair persistent TCF in 12 of 13 cases (18). Hernot et al., comparing use of rhomboid flap with use of $Z$ plasty to repair fistulas in a case series of forty patients, reported that only eight of their patients had superficial wound complications (wound infection or skin necrosis) and those eight eventually recovered by secondary healing (19). Therefore, based on our experience and these studies, not all patients requiring surgical TCF repair need general anesthesia. With careful patient selection and choice of proper surgical technique, general anesthesia can be omitted.

Although our patients did not develop subcutaneous emphysema, pneumothorax, or pneumomediastinum after TCF repair, there may be some risk of these adverse events. Therefore, a thorough preoperative survey is needed to help ensure successful surgical closure and uneventful recovery. Rennekampff et al. advocate fiberoptical visualization of the airway to preclude functional obstruction or constriction prior to performing the surgical closure of TCFs. Infections and foreign bodies should be treated well in advance of closure (20). Pulmonary function tests with measurement of the forced vital capacity is also prudent before definite closure. Geyer et al. advised preoperative overnight oximetry with the fistula occluded to identify patients who still rely on their fistula to breathe, thus avoiding the need for emergency re-cannulation (21). If these precautions are taken, then successful surgical closure can be performed 


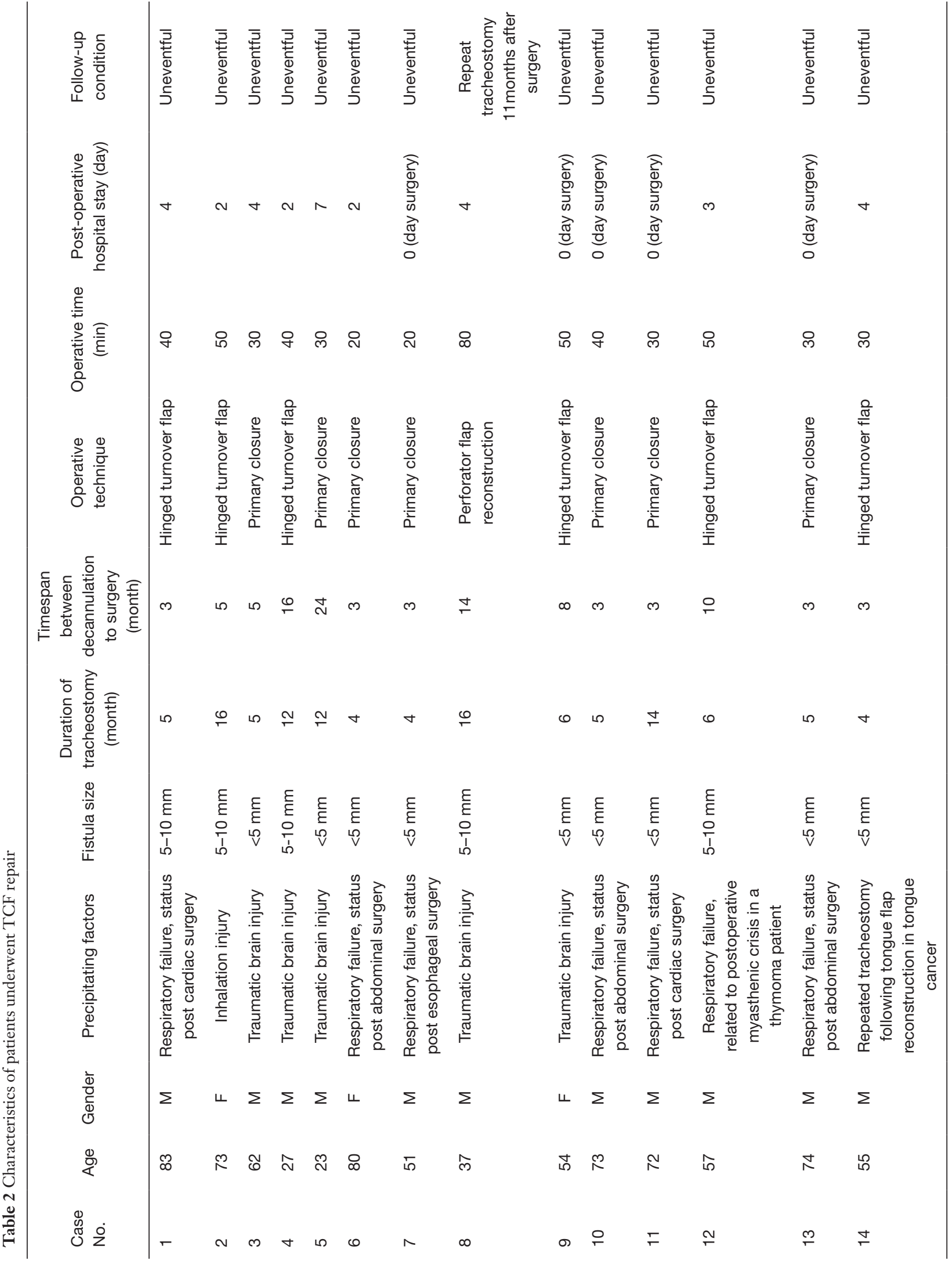




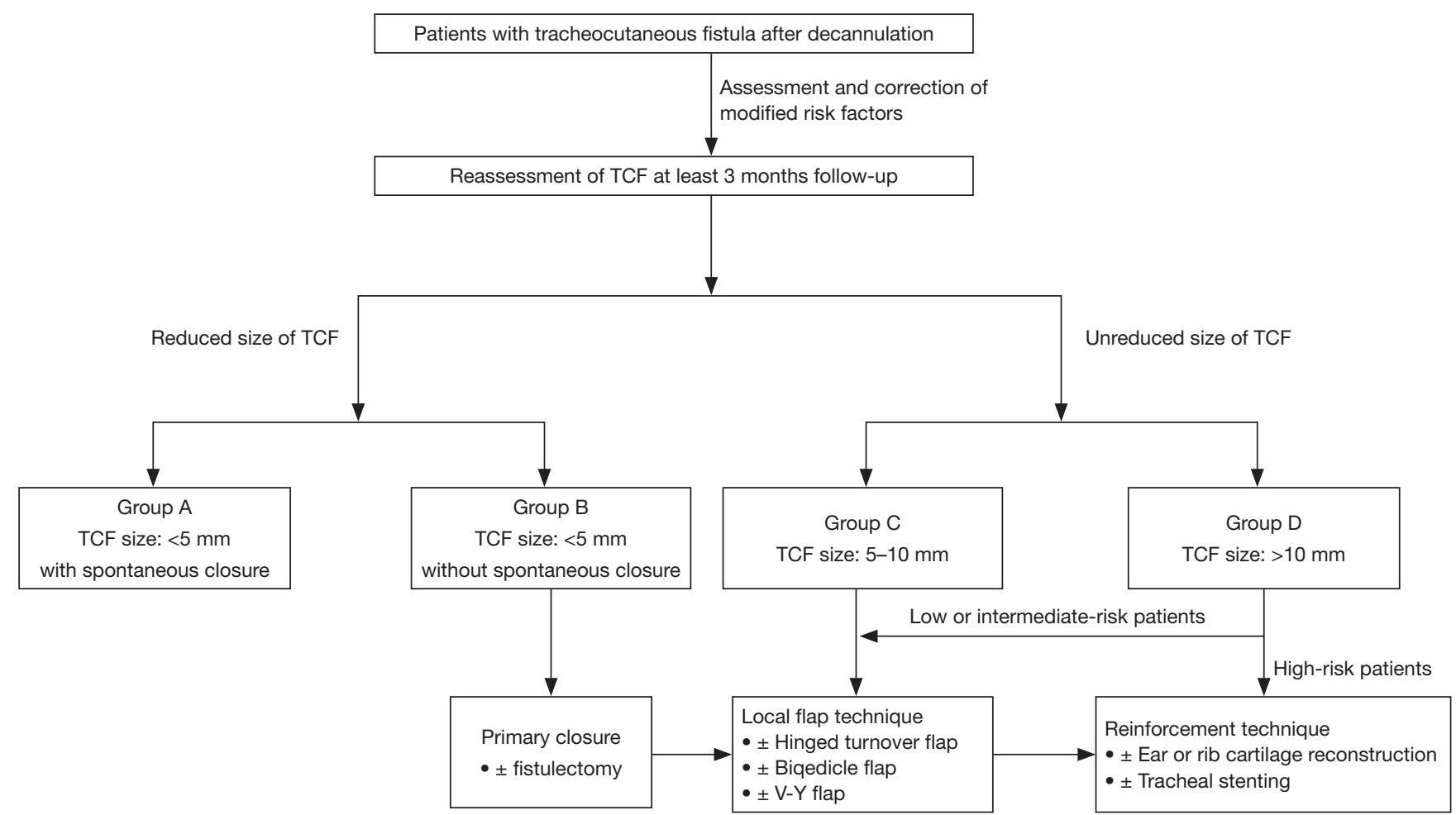

Figure 4 Decision algorithm for tracheocutaneous fistula (TCF).

safely and efficiently using the risk factor score and decision algorithm we created to for the surgical repair of TCFs.

This study has some limitations, including its retrospective design, small sample size, and selection bias when it came to choice of surgical strategy chosen for the individual patient. Further prospective studies or studies with larger sample sizes are necessary to draw firm conclusions about the optimal surgical repair of TCFs.

\section{Conclusions}

To the best of our knowledge, this study is the first to describe the management of TCF using a decision algorithm in conjunction with a modified risk scoring system. With an increasing number of patients in the intensive care units undergoing early tracheostomy, it is inevitable that incidence of TCF will continue to increase. It is hoped that outcomes can be improved with the use of this decision algorithm and appropriate surgical strategies.

\section{Acknowledgments}

Funding: This work was supported by the Ministry of
Science and Technology of Taiwan (107-2314-B-037 -063 -MY2), Ministry of Health and Welfare (MOHW107TDU-B-212-123006), Kaohsiung Medical University Hospital (KMUH107-7R26), and Kaohsiung Medical University Research Center Grant (KMU-TC108A02).

\section{Footnote}

Conflict of Interest: The authors have no conflicts of interest to declare.

Ethical Statement: The authors are accountable for all aspects of the work in ensuring that questions related to the accuracy or integrity of any part of the work are appropriately investigated and resolved. This study was approved by the institutional review board of Kaohsiung Medical University Hospital (approval number: KMUHIRB-E(I)-20190146).

Open Access Statement: This is an Open Access article distributed in accordance with the Creative Commons Attribution-NonCommercial-NoDerivs 4.0 International License (CC BY-NC-ND 4.0), which permits the non- 
commercial replication and distribution of the article with the strict proviso that no changes or edits are made and the original work is properly cited (including links to both the formal publication through the relevant DOI and the license). See: https://creativecommons.org/licenses/by-nc-nd/4.0/.

\section{References}

1. Delaney A, Bagshaw SM, Nalos M. Percutaneous dilatational tracheostomy versus surgical tracheostomy in critically ill patients: a systematic review and meta-analysis. Crit Care 2006;10:R55.

2. Pallua N, Wolter TP. Defect classification and reconstruction algorithm for patients with tracheostomy using the tunneled supraclavicular artery island flap. Langenbecks Arch Surg 2010;395:1115-9.

3. Kulber H, Passy V. Tracheostomy closure and scar revisions. Arch Otolaryngol 1972;96:22-6.

4. Eaton DA, Brown OE, Parry D. Simple technique for tracheocutaneous fistula closure in the pediatric population. Ann Otol Rhinol Laryngol 2003;112:17-9.

5. Keenan JP, Snyder GG 3rd, Lehmann WB, et al. Management of tracheocutaneous fistula. Arch Otolaryngol 1978;104:530-1.

6. Bressler KL, Kaiser PC, Dunham ME, et al. Primary closure of persistent tracheocutaneous fistula in children. Ann Otol Rhinol Laryngol 1994;103:835-7.

7. Kamiyoshihara M, Nagashima T, Takeyoshi I. A novel technique for closing a tracheocutaneous fistula using a hinged skin flap. Surg Today 2011;41:1166-8.

8. Tatekawa Y, Yamanaka H, Hasegawa T. Closure of a tracheocutaneous fistula by two hinged turnover skin flaps and a muscle flap: A case report. Int J Surg Case Rep 2013;4:170-4.

9. Lee UJ, Goh EK, Wang SG, et al. Closure of large tracheocutaneous fistula using turn-over hinge flap and $\mathrm{V}-\mathrm{Y}$ advancement flap. J Laryngol Otol 2002;116:627-9.

10. Riedel F, Reinhart Goessler U, Grupp S, et al. Management of radiation-induced tracheocutaneous tissue defects by transplantation of an ear cartilage graft and

Cite this article as: Kao CN, Liu YW, Chang PC, Chou SH, Lee SS, Kuo YR, Huang SH. Decision algorithm and surgical strategies for managing tracheocutaneous fistula. J Thorac Dis 2020;12(3):457-465. doi: 10.21037/jtd.2020.01.08 deltopectoral flap. Auris Nasus Larynx 2006;33:79-84.

11. Caronia FP, Fiorelli A, Santini M, et al. A persistent tracheocutaneous fistula closed with two hinged skin flaps and rib cartilage interpositional grafting. Gen Thorac Cardiovasc Surg 2016;64:625-8.

12. Yawn RJ, Yawn JR, Gelbard A, et al. Tracheocutaneous fistula repair with autologous auricular cartilage cap graft. Laryngoscope 2016;126:2085-8.

13. Tasca RA, Clarke RW. Tracheocutaneous fistula following paediatric tracheostomy--a 14-year experience at Alder Hey Children's Hospital. Int J Pediatr Otorhinolaryngol 2010;74:711-2.

14. Osborn AJ, de Alarcon A, Hart CK, et al. Tracheocutaneous fistula closure in the pediatric population: should secondary closure be the standard of care? Otolaryngol Head Neck Surg 2013;149:766-71.

15. Maroz N, Simman R. Wound Healing in Patients With Impaired Kidney Function. J Am Coll Clin Wound Spec 2014;5:2-7.

16. Oryan A, Alemzadeh E, Moshiri A. Role of sugar-based compounds on cutaneous wound healing: what is the evidence? J Wound Care 2019;28:s13-24.

17. Royer AK, Royer MC, Ting JY, et al. The use of a prefabricated radial forearm free flap for closure of a large tracheocutaneous fistula: a case report and review of the literature. J Med Case Rep 2015;9:251.

18. Khaja SF, Fletcher AM, Hoffman HT. Local repair of persistent tracheocutaneous fistulas. Ann Otol Rhinol Laryngol 2011;120:622-6.

19. Hernot S, Wadhera R, Kaintura M, et al. Tracheocutaneous Fistula Closure: Comparison of Rhomboid Flap Repair with Z Plasty Repair in a Case Series of 40 Patients. Aesthetic Plast Surg 2016;40:908-13.

20. Rennekampff HO, Tenenhaus M. Turnover flap closure of recalcitrant tracheostomy fistula: a simplified approach. Plast Reconstr Surg 2007;119:551-5.

21. Geyer M, Kubba H, Hartley B. Experiences of tracheocutaneous fistula closure in children: how we do it. Clin Otolaryngol 2008;33:367-9. 